\title{
Sophie Ceguré
}

Université Paris Diderot Paris 7 - ICT

\section{Les films dans le patrimoine spolié entre Est et Ouest de 1939 à la fin de la guerre froide. Une histoire en chantier}

Les saisies et spoliations de biens culturels par les nazis et les gouvernements collaborateurs pendant la Seconde Guerre mondiale, puis leur restitution ou leur rétention ne sont devenues sujets d'histoire que depuis la fin des années 1990. Ce long silence était lié aux deuils, au refoulement du passé, aux mémoires rivales de la guerre, mais aussi à l'absence de classement ou à la fermeture des archives nationales, comme à l'absence de contacts institutionnels entre les «blocs » de l'Ouest et de l'Est sur ces sujets. La fin des régimes communistes a fait émerger, médiatiquement, diplomatiquement puis scientifiquement, la question des archives ou des œuvres d'art conservées au secret à l'Est du rideau de fer. En partie par ricochet, en partie suivant d'autres dynamiques nationales ou internationales dont témoigne en 2016 l'identification d'un Modigliani spolié dans l'affaire des Panama Papers, l'actualité de l'identification du patrimoine spolié et de l'indemnisation des familles ne se dément pas depuis une vingtaine d'années, engageant des acteurs étatiques, des associations et des individus - chercheurs, généalogistes, avocats, descendants de spoliés.

La perspective croisée d'histoire du patrimoine et de la législation internationale le concernant d'une part, et d'histoire des violences et des réparations de guerre de l'autre, a été longtemps centrée sur l'enjeu majeur, écrasant, de la persécution antisémite. Elle s'élargit depuis quelques années à des recherches portant sur d'autres logiques politiques et stratégiques des spoliations. La chute du mur de Berlin a permis de déplacer le regard de l'Europe occidentale vers l'Europe centrale, 
orientale et russe. Enfin, une perspective temporelle élargie à un long après-guerre ouvre à une histoire comparée reconfigurant les notions mêmes de "spoliation» et « restitution», notamment du point de vue de leur légitimité. Les pays de l'Axe comme les États européens occupés par l'Allemagne nazie ont été, en effet, pour la plupart, successivement les victimes et les acteurs de saisies de biens culturels, puis de restitutions ou de dissimulations de ces patrimoines déplacés, sans que cette histoire ait été mise en perspective par l'une ou l'autre des mémoires nationales ou transnationales de la guerre.

Une vaste bibliographie traite désormais des tableaux, des archives, des livres spoliés dans toute l'Europe, même si l'Europe occidentale et les " territoires de l'Est » restent rarement embrassés d'un même regard. Les photographies et les films demeurent beaucoup moins connus, pour des raisons sur lesquelles il s'agit de s'interroger. D'une part, bien sûr, la dispersion et la fragilité des fonds demanderaient un travail d'inventaire analogue à celui qui a été mené pour les archives par Patricia Kennedy Grimsted depuis des années ${ }^{1}$. Les films sont un cas particulier, puisque leur lecture demande le passage par un dispositif technique médiatisant la consultation et l'identification, induisant un oubli plus rapide encore que celui des tableaux ou des papiers aisément visibles, auquel s'ajoute le plus souvent la destruction ou la dispersion des archives papier concernant leur production et leur diffusion. Enfin, la problématique des usages publics de l'image fixe et animée articule de manière particulière les enjeux stratégiques, politiques, culturels et économiques du destin d'autres biens spoliés, bien souvent étudiés dans un cadre national. Or les pratiques de saisies, de sauvetage et d'usage de ces derniers ne peuvent être compris que par les circulations transnationales de politiques qui se répondent dans l'Europe en guerre. L'essai que nous proposons n'a d'autre ambition que de tracer les grandes lignes d'un paysage historiographique encore très fragmentaire concernant les films spoliés dans l'histoire plus large du patrimoine en temps de guerre et d'après-guerre².

\section{Les films dans les pillages et spoliations nazies}

Le terme même de " trophées ", abandonné progressivement en France alors qu'il est resté d'usage à l'Est, les références fréquentes tant chez les Allemands que chez les Russes aux pillages napoléoniens, invitent à replacer la question dans l'histoire

1 Cf. sa bibliographie complète sur $<$ http://socialhistory.org/en/russia-archives-andrestitution/bibliography>, consulté le 01.02.2017.

2 Cf. l'apport précieux des travaux du projet « Le cinéma en Union soviétique et la guerre, 1939-1949 » soutenu par l'Agence nationale de la recherche (2012-2016) et dirigé par Valérie Pozner et Alexandre Sumpf. Nous nous attacherons dans le cadre de cet article essentiellement aux travaux anglophones, russophones et francophones. 
longue des spoliations culturelles et du droit international les concernant. Les pratiques de saisie, de sauvetage et d'usage des biens spoliés s'inscrivent dans la longue durée mais prennent aussi un sens nouveau au $\mathrm{XX}^{\mathrm{e}}$ siècle et particulièrement pendant le Second conflit mondial. On peut distinguer analytiquement deux grands principes d'explication concernant plus particulièrement les films, qui peuvent avoir été mobilisés par les mêmes acteurs, mais aussi avoir guidé des hommes et des organismes rivaux : une logique militaire et stratégique de conquête, et une logique d'occupation à plus long terme, dans laquelle les enjeux économiques, idéologiques et patrimoniaux de la construction d'une Europe nouvelle s'entremêlent.

\subsection{Une logique stratégique et militaire (1940-1941)}

Les biens culturels étaient devenus l'objet de pillages, et des outils de diplomatie et d'administration de la conquête dans la longue durée des conflits occidentaux. Les guerres menées par Napoléon avaient donné un tournant tant quantitatif que qualitatif à ces pratiques de saisies d'œuvres d'art et d'archives, justifiées par un projet impérial et civilisationnel (Savoy 2003). Les circulations accrues de biens culturels dans les guerres européennes ou coloniales, les questions de propriété posées à nouveaux frais par les redéfinitions de frontières à l'ère des Etats-nations font naître une réflexion dans le cadre de l'élaboration du droit international de la guerre (Sandholz 2010 ; Kecskeméti 2012). Soulignons l'importance de la deuxième conférence sur la Paix réunie à La Haye, aux Pays-Bas, en 1907. Elle aboutit à la signature d'une Convention « concernant les lois et coutumes de la guerre sur terre ». Cette convention protège les populations civiles au moment des combats. L'article 35 de l'annexe à la Convention limite le droit de saisie aux seuls « biens mobiliers pouvant servir aux opérations de la guerre ». L'article 56 stipule que « les biens des communes, ceux des établissements consacrés aux cultes, à la charité et à l'instruction, aux arts et aux sciences, même appartenant à l'État, seront traités comme la propriété privée. Toute saisie, destruction ou dégradation intentionnelle de semblables établissements, de monuments historiques, d'œuvres d'art et de science, est interdite et doit être poursuivie ».

Les destructions patrimoniales de la Première Guerre mondiale, puis les menaces de la guerre aérienne sur les musées et les monuments pendant la guerre civile espagnole ont pour conséquence des réflexions menées dans les cadres nationaux, aboutissant à la préparation de plan d'évacuation et de protection anticipant un nouveau conflit. Toute l'Europe anticipe et redoute un nouveau conflit mondial. Ces évacuations bien organisées en France, surtout pour les musées et les Archives nationales et départementales puis de plus en plus improvisées, n'éviteront pas de nombreuses destructions hâtives par le feu. A l'inverse, l'URSS, qui vivait depuis des années dans le climat d'encerclement et d'agression capitaliste, ne prépare pas ses archives au conflit. Le dogme stratégique de l'inviolabilité du territoire avait 
largement stérilisé la réflexion (Bellamy 2008 ; Kopylova 1990 ; Cœuré 2015). Quant au droit international, il ne progresse guère, et c'est toujours la convention de La Haye qui sera en vigueur en 1939-1945.

Quand la guerre revient en Europe en 1939, ce sont tout d'abord des pillages purs et simples qui sont menés, à la bonne fortune des combats. C'est le cas tant dans les Républiques baltes et dans la partie de la Pologne occupées par l'Armée rouge (Fomin 2005 : 625) puis en Pologne annexée par les Nazis en 1939, enfin lors de l'avancée des troupes allemandes en Belgique et en France pendant la "Guerre éclair » du printemps 1940. Des chartes anciennes disparaissent ainsi des châteaux de la Loire. Des péniches ou des trains arrêtés par la débâcle de l'armée française en mai-juin 1940, des châteaux utilisés comme dépôts d'évacuation sont visités à l'occasion et font l'objet de belles trouvailles par la Wehrmacht. Parmi les découvertes les plus précieuses, un wagon de documents du Grand Quartier Général du ministère de la Guerre est saisi par hasard, bloqué en gare de la Charité-sur-Loire, prise que les Allemands allaient qualifier de « sensationnelle découverte » (Cœuré 2013). Fondée en 1915, la Section cinématographique de l'Armée (SCA) avait accumulé plus de 2000 films tournés pendant la Première Guerre mondiale, et repris en 1939-1940 le tournage de films d'instruction, «d'action morale » et de propagande. Sans consigne d'évacuation ni de protection des documents, le service se replie en Indre-et-Loire, puis dans le Lot-et-Garonne. Une partie des films positifs disparaissent alors, tandis que les négatifs demeurés à Tours sont saisis en octobre 1940 (Launey 2008 ; Brunel 2014). Avec eux sont emportés des films que la Cinémathèque française, dirigée par Henri Langlois depuis sa fondation en 1936, avait évacués de ses dépôts du Trocadéro et du musée de l'Homme et confiés au SCA (Mannoni 2006).

Le ministère des Affaires étrangères allemand est le premier à organiser un « commando » subordonné à la police militaire de campagne et dirigé par Eberhard von Künsberg. Le " Soko K », rapide et motorisé, perquisitionne dès les premières victoires de la campagne en Hollande, en Belgique et en France. Il visite les ministères français -Affaires étrangères, Guerre, Colonies, etc. - dès le 14 juin1940, en parallèle avec d'autres commissions spéciales d'archivistes, envoyés par les Archives militaires (Heeresarchiv) ou par la Kriegsmarine. Les Allemands s'appuient sur une interprétation extensive de la convention de la Haye pour justifier les saisies, et imposent leurs vues aux jurisconsultes de Vichy. Il s'agit en fait bien davantage de futures archives, de documents administratifs et non historiques, pour la plupart récents et encore d'usage courant. Ils sont considérés par les Allemands comme nécessaires à la conduite politique et militaire de la guerre et quantitativement, constituent l'essentiel des documents emportés depuis la France. On y trouve les papiers et les bibliothèques de nombreux ministères, les dossiers de la police et du contre-espionnage. On y trouve également de nombreux documents diplomatiques, y compris des documents plus symboliques qu'opérationnels, tel l'original du traité de Versailles signé par 
tous les participants à la conférence de la Paix, qui est envoyé aussitôt par avion à Hitler comme un trophée de guerre. Au même moment, un commando du RSHA (Reichssicherheitshauptamt), Office central de sécurité du Reich, dirigé par Helmut Knochen, docteur en philosophie et ancien journaliste membre du NSDAP, passe par la Belgique puis perquisitionne la préfecture de police de Paris. Il s'empare également par exemple des dossiers de la Fédération internationale des Archives du Film (FIAF), dont le siège était au Palais-Royal. La dynamique de conquête est relancée au moment de l'invasion de la zone sud française en 1943, ou quand les Allemands prennent pied en Tunisie et se saisissent des fonds de la Sûreté générale.

L'avancée rapide des troupes allemandes vers l'Est après la rupture du pacte germano-soviétique en juin 1941 représente le dernier moment clé de cette logique stratégique et militaire. Quelques heures après le déclenchement du plan Barbarossa, l'URSS organise à la hâte l'évacuation. Bien davantage qu'en France, le recul militaire s'accompagne de destructions massives de documents, et les évacuations désordonnées causent des pertes énormes. Le « Soko K » intervient de nouveau et se saisit de documentation stratégique dans les pays baltes, en Ukraine et en Biélorussie. Le butin des troupes nazies est alors considérable avec des documents qui forment pour certains, comme en France, des trophées symboliques autant qu'utiles. Les principaux sont les archives du Parti communiste, que les Soviétiques n'avaient pas eu le temps d'évacuer de Smolensk et de Dniepropetrovsk (Dmitrieva et al. 2005).

Dans la partie occidentale de l'Europe tout au moins, on reste là dans une image de la guerre « correcte » diffusée en 1940, ou du moins respectant le droit international, à laquelle s'accrocheront dans leurs mémoires et leurs procès les responsables allemands des spoliations culturelles. Il leur faudra pour ceci passer sous silence le déplacement vers Berlin d'archives historiques, privées ou publiques, comme par exemple des fonds du ministère français de la Guerre depuis le XVII e siècle, des archives du ministère de l'Intérieur depuis le XIX ${ }^{e}$ siècle, ainsi que des films du SCA datant de la Première Guerre mondiale. On n'est plus là dans le registre de la saisie légale, mais bien dans celui de la spoliation.

\subsection{Une logique d'occupation : la construction $d u$ "Reich de mille ans "}

L'évacuation des biens culturels avait été d'abord destinée à les protéger des combats et des bombardements. En revanche, l'anticipation de l'installation durable de l'occupant allemand et de l'usage de documents politiques courants par l'occupant reste très tardive, voire inexistante. Et si le précédent des autodafés de livres par les Nazis avait été très médiatisé, peu nombreux sont ceux qui peuvent prévoir qu'une lutte idéologique, culturelle et même historique allait être menée sur le sol européen, même parmi les émigrés allemands qui avaient cherché asile contre Hitler en France ou en URSS. 
La reconstruction d'une Europe nazifiée comprend une dimension culturelle bien connue, surtout pour l'Europe de l'Ouest (Corcy 2005 ; Karlsgodt 2011) où elle est menée par les administrations miliaires mais où elle prend également une dimension plus subtile de gestion des gouvernements collaborateurs. À l'Est se déploie dans une brutalité nue un régime d'occupation, colonial et génocidaire (Voisin 2015 : 61 et ss.). Partout il s'agit, on le sait, de gagner la guerre tout en commençant à épurer l'Europe des ennemis du Reich - démocrates, communistes, juifs, francs-maçons. La spoliation économique s'accompagne alors de l'étude de la culture des peuples promis à la destruction ou à la soumission pour contrer leur influence intellectuelle et culturelle - et légitimer a posteriori ces politiques.

L'ERR, Einsatzstab Reichsleiter Rosenberg, est l'acteur majeur, et sans doute le plus connu, de la spoliation culturelle. Proche d'Hitler, Alfred Rosenberg avait été chargé de l'idéologie et de " la formation et l'éducation intellectuelles et morales » en 1934 au sein du parti nazi, le NSDAP. Par son expérience personnelle - né dans les régions baltes de langue allemande de l'Empire russe, il avait été témoin de la révolution d'Octobre à Moscou - comme par ses publications, Rosenberg avait joué un rôle important dans la définition de la conspiration « judéo-bolchevique » et « judéo-maçonnique » contre l'Allemagne. Il obtient d'Hitler un « droit de perquisition dans les bibliothèques, loges et autres institutions scientifiques et culturelles », puis étend son action à l'Europe orientale et à l'URSS en devenant ministre du Reich pour les territoires occupés de l'Est, où il organise l'assassinat de centaines de milliers de Juifs (Heuss 2000 ; Grimsted 2016). Rosenberg y rivalise avec Künsberg, dont les commandos finissent par être dissous en 1943. Il doit composer avec les visées spoliatrices d'Herman Goering quand il s'empare des collections d'œuvres d'art appartenant à des juifs dans toute l'Europe, avec les objectifs plus policiers du RSHA et de la Gestapo, et avec la politique de propagande de Goebbels (Dreyfus 2015).

C'est ainsi que les principaux organismes du judaïsme français comme l'Alliance israélite universelle, de la franc-maçonnerie (le Grand Orient, la Grande Loge de France), les grandes collections de Judaica des pays baltes, d'Ukraine et de Biélorussie, mais aussi des institutions liées à la Ligue des droits de l'homme, des partis et organisations syndicales de gauche, ainsi que d'innombrables galeries d'art ou logements privés de personnalités désignées comme juives et/ou connues pour leur engagement antinazi sont visités entre 1940 et 1944, parfois successivement par ces organisations rivales. Cette politique - saisir, détruire, mais aussi documenter le patrimoine culturel de l'ennemi pour les générations aryennes à venir - n'est menée en France, en Belgique ou au Luxembourg qu'aux dépens des personnes ou associations privées, alors qu'elle s'attaque en parallèle, dans l'URSS occupée, au patrimoine 
d'État ${ }^{3}$. Les livres et manuscrits anciens de Kiev, Voronej ou Rostov, d'innombrables tableaux et statues sont emportés, la fameuse «Chambre d'ambre » du palais de Tsarskoe Selo démontée et envoyée à Koenigsberg en 1941.

C'est dans le domaine du cinéma que les objectifs de domination économique, de propagande et d'épuration culturelle sont les plus étroitement liés (Vande Winkel $\&$ Welch 2011). Il s'agit de récupérer la cellulose et les sels d'argent des films saisis, d'« aryaniser » les cinémas et les compagnies de production, de subordonner les industries nationales, française belge ou tchécoslovaque et d'éradiquer l'influence anglo-américaine dans la production et la distribution au profit des firmes allemandes. La refondation d'une Chambre internationale du film en juillet 1941 (IFK), la création de la compagnie Continental film basée à Paris, s'accompagnent aussi d'une politique systématique de censure confiée à la Filmprüfstelle. Elle délivre les visas d'exploitation des films après censure, établit des listes de films juifs ou « bolcheviques », saisit le fonds du studio Albatros fondé à Montreuil par des Russes blancs dans les années 1920. Elle installe à Courbevoie une véritable cinémathèque pour visionner systématiquement les films du SCA qui n'avait pas déjà été emportés à Berlin.

L'action du major Frank Hensel, membre du NSDAP, ancien directeur de la Reichsfilmarchiv, président de la Fédération internationale des archives du film (FIAF) fondée en 1938, s'inscrit dans cette politique globale et permet de s'interroger sur les ambiguités des accommodations et des collaborations dans l'Europe occupée. Ce parfait francophone parcourt l'Europe pour récupérer, légalement par l'intermédiaire notamment d'achat en Suède et au Portugal neutres, ou en s'en emparant tout simplement, les films étrangers qui l'intéressent, et les envoyer à Berlin. Concurrent du ministère de la Propagande, il entretient une relation ambiguë avec des Français qu'il connaît de longue date comme Henri Langlois et Georges Franju (Aurich 2002 ; Barbin 2005). Comme les archives, publiques ou privées, comme les tableaux, les films, surtout américains ou soviétiques, font alors en France l'objet de tentatives de sauvetage et de dissimulation, qui profitent des rivalités entre les organismes de Vichy et les Allemands. Ces efforts seront mis en avant sans surprise après la Libération.

Le projet de cinémathèque européenne de Frank Hensel ou de musée d'art européen en Ukraine, l'idée personnelle d'Hitler de créer à Linz un grand musée rassemblant les œuvres d'art de toutes les zones occupées, l'occupation du quai d'Orsay et du ministère de la Guerre pendant quatre ans par des archivistes et historiens allemands qui font réaliser et envoyer à Berlin des milliers de photographies de documents, montrent que

Pour une analyse des usages politiques et idéologiques des archives et de la logique comparée des spoliations entre le Troisième Reich et l'URSS, je me permets de renvoyer à mon ouvrage, La mémoire spoliée (Cœuré 2013), chapitre 5 « La guerre, le droit, la nation, la mémoire ». 
ces saisies et ces spoliations ne sont pas seulement immédiatement prédatrices, mais déploient une réflexion sur l'avenir d'une Europe durablement organisée par le Reich et d'une histoire réécrite en conformité avec les principes nationaux-socialistes. Il s'agit par exemple de rassembler physiquement à Berlin tout le patrimoine culturel national, toutes les archives mais aussi les livres, les œuvres d'art, les matériaux archéologiques, désignés comme " germaniques » et justifiant la conquête par le Reich d'une partie de l'espace européen. Le travail scientifique de recherche et de constitutions de listes préliminaires à de futures saisies concerne particulièrement la France, dans l'optique voulue par Goebbels de préparer un traité de Paix qui effacerait en quelque sorte l'histoire de l'Europe depuis les traités de Westphalie, mais aussi les pays baltes dont les archives hanséatiques sont "rapatriées », la Pologne et l'URSS (Stein 2012). Il est mené en même temps que des matériaux commencent à être collectés illégalement dans toute l'Europe, soit ouvertement, soit sous couvert d'organismes de "protection » (Kuntschutz, Archivschutz, etc.). Enfin, alors que les galeries et collectionneurs juifs sont systématiquement spoliés, un vaste chantier de documentation photographique des œuvres d'art et monuments historiques français est mené sous l'égide du Bildarchiv Foto Marburg, centre national allemand de documentation pour l'histoire de l'art et de l'architecture par le Kunsthistorische Forschungsstätte Paris, premier institut allemand d'histoire de l'art en France, créé au début de l'année 1942, dans le bâtiment spolié de l'ancienne ambassade de Tchécoslovaquie, au 18 rue Bonaparte ${ }^{4}$.

\subsection{Les usages nazis du patrimoine spolié}

Qu'ont fait les dirigeants du IIIe Reich du patrimoine spolié, dans le peu de temps qui leur restait en réalité avant la défaite militaire ? Dans l'énorme masse d'archives et de livres envoyés à Berlin, seuls quelques documents stratégiquement et politiquement importants commencent à être inventoriés. Des traces de lecture existent dans les archives militaires françaises ou celles de la Ligue des droits de l'homme, des fichiers commencent à être constitués à partir des archives policières et franc-maçonnes. Distillant des informations à la presse allemande et collaborationniste, le ministère des Affaires étrangères allemand publie également plusieurs recueils de documents à Berlin dans les collections « Les origines de la guerre de 1939 » et « Les documents secrets de l'État-major français ». C'est dans ce cadre que les rapports de Jean Herbette, ambassadeur de France en URSS dans les années 1920, sont traduits en 1943 à Paris sous le titre de Un diplomate français parle du péril bolchevique afin de

Cf. le projet de recherche mené par l'Institut historique allemand sur : $<$ https://dfk-paris. org/fr/research-project/entre-art-science-et-politique-d\%E2\%80\%99occupation-1211. html>, consulté le 01.02.2017. 
« déchirer le voile de mensonges dont se recouvraient les plans du Kremlin » (Cœuré 2013). Les films saisis dans les pays conquis sont analysés sur un plan technique, idéologique et militaire. La section cinéma du ministère de la Propagande détourne et remonte des extraits d'actualités américaines pour «démasquer » Roosevelt et la « ploutocratie juive » (Crips, Férard \& Gabriel 2014). La propagande nazie et vichyste souhaite de même mobiliser les sources d'archives authentiques, au fil de grandes expositions historiques visant un large public. C'est ainsi que des documents, objets et photographies saisis dans les loges sont utilisés pour l'exposition « La franc-maçonnerie dévoilée » tenue au Grand Palais en 1941 puis circulant en France. Les trophées de guerres sont donc mobilisés parallèlement à l'usage de la fiction de propagande dans des films tels que Les Rothschilds (UFA 1940) ou Forces occultes (Nova Film, commandité par la Propaganda-Abteilung Frankreich, 1943). Enfin, les studios de Kiev et de Riga sont occupés pour produire des films de propagande nazie. À Riga, ceux-ci utilisent parfois des images de la première occupation soviétique menée en 1939-1941 à la suite du pacte Hitler-Ribbentrop - pour dénoncer la politique bolchevique ${ }^{5}$.

On sait qu'Herman Goering avait commencé à créer son musée privé dans sa propriété de Carinhall au Nord de Berlin. Le siège du Reichsfilmarchiv et de la Direction du cinéma de Vichy, installés au 7 rue de Messine dans un immeuble spolié à une famille juive, est en avril 1944 le théâtre d'une scène apparemment paradoxale : la projection privée pour Frank Hensel de Tempête sur l'Asie du réalisateur soviétique Poudovkine, en présence de Jean Cocteau (Barbin 2005). Mais dans l'ensemble, les dignitaires nazis ne profitent guère des œuvres d'art spoliées, entassées à Berlin dans des entrepôts, des bâtiments confisqués à des francs-maçons ou à des juifs, à Dresde pour le futur musée de Linz, à Francfort pour la future bibliothèque de la « Hauteécole » du parti nazi, parfois restés dans des wagons de train sans même être déballés. Dès 1943, les évacuations vers l'Est du Reich s'organisent sous les bombardements, principalement en Silésie et Bohême-Moravie.

Une réflexion comparée reste à conduire entre la politique du Reich et ses saisies patrimoniales et l'exploitation politique des archives menées par l'URSS en Pologne et dans les pays baltes après 1939. On sait par exemple que les listes et les fichiers de la république bourgeoise de Lettonie formèrent un matériau privilégié pour l'épuration et la déportation menée par le NKVD en 1940-1941 (Denis 2012). Certes bien différente de celle des nazis, la conception soviétique de la légalité internationale et de la finalité de la culture s'en rapprochait par sa politisation et son intrusion dans les patrimoines privés, et allait se déployer dès 1944 dans l'Europe libérée par l'Armée rouge. 


\section{Le destin des biens saisis et spoliés dans la longue guerre froide}

\subsection{Saisies, restitutions et trophées alliés}

S'appuyant à leur tour sur la convention de La Haye, et conscients des destructions systématiques par les nazis des preuves de la politique d'extermination de masse des Juifs d'Europe et de la répression concentrationnaire, les alliés convergeant vers Berlin et mènent une véritable course aux archives. Il s'agit de documenter les crimes nazis en vue de convaincre les opinions dans la guerre finissante, puis d'organiser des procès au niveau national et international. Le potentiel de la preuve par l'image fixe et animée est la grande nouveauté de cette sortie de guerre (Delage 2006 ; Pozner, Sumpf \& Voisin 2015). Les opérateurs des services cinéma et photo des armées américaine, britannique et soviétique filment les victimes, font témoigner les rescapés. Parallèlement à la recherche de preuves médico-légales, les documents papiers ou audiovisuels saisis sont envoyés dans les capitales, à Moscou, Londres ou Washington. Les Français s'emparent de bobines d'actualités allemandes trouvées dans la région de Kehl après la libération de Strasbourg. Il s'agit aussi de collecter pour comprendre à l'avenir l'histoire du national-socialisme. En 1946, le commandement français en Autriche confie à Henri Langlois dix films nazis qui viennent d'être saisis, dont le tristement célèbre Juif Süss. En 1945-1947, une mission de la Librairie du Congrès dirigée par le professeur Luther Evans parcourt l'Allemagne pour collecter la documentation nazie sensible qui sans cela aurait été détruite, emportant des archives, des livres (par exemple les 60000 volumes de la bibliothèque du Reichskolonialbund) ou des films (Dean sd).

Pendant leur progression, les soldats alliés découvrent dans le même temps les caches de biens culturels spoliés, châteaux, mines de sels, etc., évacués de Berlin. Or Staline avait préparé sa riposte aux gigantesques pillages et destructions dont l'URSS avait été la victime entre l'invasion de son territoire et le retournement militaire en sa faveur de 1943, et lancé un travail systématique d'évaluation des dommages. La «Commission d'État extraordinaire pour l'enregistrement et la recherche des crimes des occupants allemands fascistes et de leurs complices et des dommages commis par eux aux citoyens, kolkhozes, organisations publiques et institutions de l'URSS » avait commencé son travail dès 1941 (Moine 2015). La réflexion interalliée également menée avant même la fin de la guerre avait abouti à la Déclaration de Londres du 5 janvier 1943, qui condamnait les pillages, les spoliations mais aussi les ventes forcées. Le patrimoine culturel fait l'objet de l'attention particulière des anglo-américains qui créent le Monuments, Fine Arts and Archives Program (MFA\&A) : les Monument men sont parfois accompagnés sur le terrain par le Service Beaux-Arts de la Première armée française, dirigé par le peintre Jean Rigaud. Les discussions quadripartites 
continuent dans la dynamique de la libération de l'Europe et de l'occupation de l'Allemagne et de l'Autriche, initiant de nouveaux débats juridiques sur les notions de restitution et de compensation. Ils permettent la mise en place d'accords fondés sur un système de claims (Kurtz 2010).

Les organismes mis en place sous tutelle des administrations d'occupation militaire ou dans le cadre d'un retour aux relations diplomatiques bilatérales avec la Pologne et la Tchécoslovaquie, puis la RFA et la RDA permettent alors l'identification et les restitutions croisées de millions d'œuvres d'art, livres, films et archives spoliés par les nazis. Les relations entre les responsables de ces recherches, menées pour la France sous l'impulsion énergique de Rose Valland, conservatrice au Jeu de Paume sous l'Occupation (Bouchoux 2006), éclairent d'un jour différent les débuts de la guerre froide et les tensions qui s'installent avec les Soviétiques et leur « sens très particulier de la propriété » (Cœuré à paraitre). Idéologie et économie se combinent de nouveau. De fait, de nouveaux pillages ponctuels avaient accompagné l'avancée des Français, des Britanniques et Américains, ciblant tant les objets symboliques ayant par exemple appartenu à Hitler, que ceux qui sont susceptibles d'avoir de la valeur (Alford 2011). Mais seule l'Armée rouge mène une politique massive de pillages destinée à compenser les destructions et les pertes subies lors de l'occupation nazie de l'URSS, avec la couverture implicite des autorités qui autorisent par exemple l'envoi postal de leur butin par les soldats vers l'Union soviétique (Naimark 1995 ; Schechter 2015). C'est sous l'égide du Comité d'État à la Défense (GKO) que sont organisées dans le même temps des « brigades des trophées », spoliant méthodiquement les usines et les laboratoires allemands. Les musées et les bibliothèques allemands sont aussi visités par des universitaires et conservateurs sous l'uniforme. Margarita Rudomino, dont la bibliothèque des langues étrangères de Moscou porte maintenant le nom, s'empare de milliers d'ouvrages dont une célèbre Bible de Gutenberg, datant de 1455 (Akinsha \& Kozlov 1995 ; Akinsha 2010). Les studios de cinéma et le Reichsfilmarchiv sont dépouillés sous l'égide de Gueorgui Avenarius, ancien professeur d'histoire du cinéma à Kiev et à Moscou. Même si c'est surtout le matériel qui les intéresse câbles, produits, chimiques, machines à écrire, costumes, décors, etc., les Soviétiques emportent également les archives et les pellicules (Pozner 2012).

Ce gigantesque déménagement s'effectue parallèlement aux restitutions effectuées par l'URSS dans le cadre du droit international, restitutions qui s'avèrent très partielles. Car si les Français reçoivent de la zone soviétique des canons, des tapis, des tableaux et du mobilier, toutes les archives et les livres qu'ils récupèrent proviennent des zones américaine et britannique, où se trouvait il est vrai la grande majorité des dépôts nazis, mais pas tous. Les autorités militaires et diplomatiques françaises sont rapidement alertées par des informateurs sur le terrain sur le fait que des quantités importantes de documents, de livres et d'œuvres d'art spoliés par les nazis ne font pas le trajet retour vers leur patrie, mais sont au contraire envoyés à Moscou. 


\subsection{Logiques de blocs et ère du secret}

La guerre froide qui s'installe interrompt alors la circulation d'informations sur le patrimoine spolié entre les blocs, que ce soit par le biais de la diplomatie ou des organisations professionnelles comme le Conseil international des archives (créé en 1948) ou la FIAF. Les autorités soviétiques sont parfaitement conscientes qu'elles conservent dans l'illégalité des biens culturels qui avaient été spoliés par les nazis à leurs anciens alliés. C'est donc sous le régime du secret que sont constitués des « fonds spéciaux » des musées (par exemple à l'Ermitage) ou des bibliothèques, et que sont créés deux centres spécialisés pour les archives et les films. En 1946 sont fondées au nord de Moscou les Archives spéciales centrales d'Etat, dirigées directement par le NKVD puis le KGB hors du réseau archivistique officiel de l'URSS et conservant des millions de documents d'archives provenant de toute l'Europe (Cœuré 2013). Les films arrivent quant à eux au « dépôt » fondé en 1935 dans la région de Moscou, mais l'importance du fonds de films étrangers, presque cinq fois supérieur aux collections de films russes et soviétiques, entraîne en 1948 la création d'un véritable centre du film, le Gosfilmofond, destiné, à l'image des cinémathèques occidentales, à l'étude et à la conservation (Pozner 2012). C'est là que seront également déposés des films japonais saisis en Mandchourie.

À partir des années 1950, les retours se poursuivent, mais dans une logique de blocs. Les États-Unis restituent à la RFA les originaux des films documentaires ou de fictions allemands saisis en 1945 et lui remettent les archives nazies, à condition de les ouvrir largement. En revanche, Washington conserve le fonds de Smolensk, soit 536 dossiers d'archives du parti communiste qui avaient été saisies par les Allemands et qu'ils avaient découvertes à leur tour en Bavière. Ils les confient au contre-espionnage, avant de les ouvrir aux « soviétologues » universitaires. Dans les années 1960, les Archives françaises du film installées à Bois-d'Arcy reçoivent de l'ambassade de France à Washington 30 caisses de films et 23 enveloppes de fiches, ainsi qu'une trentaine de caisses contenant chacune une centaine de microfilms réalisés par les Allemands dans les Archives diplomatiques françaises (Leblay-Kinoshita \& Maure 2014). Quant au patrimoine spolié par les nazis qui se trouve désormais de l'autre côté du rideau de fer, « sauvé » par l'Armée rouge, il est restitué avec parcimonie par l'URSS après la mort de Staline aux « pays frères ». La RDA reçoit ainsi des archives et des tableaux qui avaient été transportés à Moscou, et des films qui étaient restés dans l'ancienne zone d'occupation soviétique et sur lesquels l'URSS avait gardé la haute main, ce qui permet l'ouverture en 1955, des Archives cinématographiques d'État, Staatliches Filmarchiv der DDR (Crips, Férard \& Gabriel 2014).

Rose Valland reste toute sa vie persuadée, avec raison, qu'une partie des archives et des œuvres d'art françaises se trouvent en Allemagne de l'Est, en Pologne ou en URSS. Mais en l'absence de communication entre les anciens alliés, leur localisation ressort 
désormais de la rumeur et de l'enquête informelle. Henri Langlois qui s'était rendu en vain à plusieurs reprises à Berlin à la recherche des films perdus en 1940, apprend qu'ils se trouveraient à Moscou. En 1955, il charge Anne et Gérard Philippe, qui se rendent en URSS pour une tournée théâtrale, de faire des recherches au Gosfilmofond, en vain toujours (Mannoni 2006). L'oubli s'installe peu à peu, malgré des signaux épars qui se déclenchent avec la détente. En 1966, à l'occasion du voyage du général de Gaulle à Moscou, quelques documents concernant la Résistance, ou pillés chez le célèbre écrivain français André Maurois, sont offerts en cadeau à la France, sans pour autant susciter de nouvelles enquêtes. Dans les années 1960 et 1970, l'amitié entre deux hommes, Raymond Borde, le co-fondateur et conservateur de la Cinémathèque de Toulouse et son homologue russe du Gosfilmofond à Moscou, Viktor Privato, permet la constitution à Toulouse d'un riche fonds de films soviétiques, mais aussi de westerns américains issues des «trophées » pillés par les Allemands. Borde récupère également le négatif nitrate original du film de Jean Renoir La Grande Illusion, qui n'était connu depuis l'après-guerre que par divers contretypes français, allemands et américains (Gauthier \& Laurent 2012).

\subsection{Le destin des trophées}

Le patrimoine dispersé ou saisi qui rentre « à la maison », que ce soit à l'Est ou à l'Ouest, fait l'objet d'un travail de réintégration dans les collections, de restitution aux spoliés, ou d'attribution quand ceux-ci ont disparu dans la guerre. La reconstitution des fonds d'archives, effectuée par exemple au quai d'Orsay à partir des doubles conservés dans les postes à l'étranger, signifie aussi l'effacement des traces de la spoliation, et donc de la défaite et de la collaboration. Progressivement, ce phénomène des saisies, des spoliations et des trophées tombe dans l'oubli, pour tout un ensemble de raisons. Les procès internationaux sont centrés sur les pertes humaines et les dommages économiques, et même si une journée du procès de Nuremberg est consacrée au patrimoine culturel, l'épuration ignore ses acteurs. L'histoire heurtée des tableaux ou meubles spoliés en France, non identifiés dans un premier temps et estampillés « Musées nationaux récupération » est éclairante (Bouchoux 2013). Si les images d'actualité nazies continuent à être largement utilisées des deux côtés du Rideau de fer (Pozner 2015), leur histoire n'est plus connue. Pratiqué systématiquement au Gosfilmofond, le transfert sur support " safety » des éléments nitrate récupérés en 1945 par les Soviétiques, et la destruction des supports originels conduisent ainsi à un effacement des marques de la provenance sur la pellicule (marque, type, laboratoire) et entraînent la destruction des fiches les concernant. Il faut relier ces pratiques aux cheminements du deuil et de la reconstruction nationale après la défaite nazie, qui conduisent à des refoulements de la défaite militaire et des persécutions antisémites (Rousso 2001 ; Salomoni 2007). Le droit international de protection du patrimoine en 
tant de guerre et de restitution des biens culturels faisant l'objet de contentieux entre États progresse sous l'égide de l'UNESCO, mais son application reste freinée par la Guerre froide et la décolonisation.

En URSS, le secret d'État empêche toute valorisation des œuvres d'art, des livres et des archives « deux fois spoliés » (Grismsted 2002 ; 2013). L'usage " opérationnel » des archives allemandes, polonaises ou françaises pour le renseignement militaire ou politique perd progressivement de son intérêt au fur et à mesure que le temps passe. Seuls certains films trophées connaissent une paradoxale deuxième vie. La quasiinterruption de la production de films soviétiques après dix années de censure et de guerre conduit le Comité d'État du cinéma soviétique à faire appel aux ressources de films américains, allemands, mais aussi dans une moindre mesure italiens et français. Doublés, remontés, projetés sans générique afin d'effacer leur origine spoliée, ces films connaissent parfois un succès colossal. Par leur langage cinématographique, l'image de la société de consommation, du vêtement, de l'érotisme qu'ils transmettent aux spectateurs soviétiques, ces films trophées ont sur l'occidentalisation de l'Union soviétique des effets qui commencent à peine à être étudiés (Pozner 2012). «La seule série des Tarzans a probablement plus fait pour la déstalinisation que tous les discours de Khrouchtchev au $\mathrm{XX}^{\mathrm{e}}$ congrès et après » écrira Joseph Brodsky dans " Les trophées ", très beau texte de ses souvenirs de jeunesse à Leningrad écrit directement en anglais depuis son exil américain (Brodsky 1988).

La France sera moins efficace dans l'effacement des traces, même elle parvient à gérer les procès que tentent les Majors américaines pour récupérer les films saisis par Vichy ou les Allemands et déposés à la Cinémathèque. En juillet 1952 est projeté au Quartier latin le film La Ville dorée de Veit Harlan, tourné pendant l'occupation nazie et présentant Prague comme une ville allemande dans une nation tchèque " dégénérée », récupéré dans le séquestre d'une ancienne société allemande. La protestation de l'ambassade de Tchécoslovaquie en France conduit à son retrait rapide des écrans ${ }^{6}$.

L'histoire du patrimoine spolié et déplacé reste à écrire dans la perspective des usages et de l'oubli de ces biens culturels. Les « films trophées », documentaires ou de fiction sont, du fait de leurs usages partiels, ceux qui s'inscrivent le plus ouvertement dans l'histoire complexe des circulations politiques et culturelles entre l'Allemagne nazie et ses adversaires, puis entre l'Est communiste et l'Ouest capitaliste, tandis que les archives, les livres et les œuvres d'art demeuraient largement du domaine du secret et de l'oubli. Il faut attendre la chute du mur de Berlin et la disparition de l'URSS pour que s'installe dans les années 1990-2000 une dynamique de restitutions des archives 190 Relations culturelles avec la France et les autres pays 1952-1954, pp. 77, 88, 104. 
vers la France, la Belgique ou les Pays-Bas par la Fédération de Russie, mais qui ne concerne pas les anciennes démocraties populaires et n'inclut - pas encore ? les livres, les œuvres d'art ou les films. La dynamique des contacts entre chercheurs et entre institutions - entre le Gosfilmofond et les Archives françaises du film par exemple - permet tout au moins un partage des connaissances sur ces étonnantes circulations de biens spoliés pendant la Seconde Guerre mondiale et la guerre froide.

\section{Renvois bibliographiques}

Akinsha Konstantin, « Stalin’s Decrees and Soviet Trophy Brigades : Compensation, Restitution in Kind, or "Trophies" of War ? », International Journal of Cultural Property, 2010, $\mathrm{n}^{\circ} 17$, pp. 195-216.

Akinsha Konstantin, Grigorii Kozlov, Beautiful loot. The Soviet Plunder of Europe's Art Treasures, New York : Random House, 1995.

Alford Kenneth, Allied Looting in World War II : Thefts of Art, Manuscripts, Stamps and Jewelry in Europe, Jefferson, NC : McFarland \& Co, 2011.

Barbin Pierre, La cinémathèque française, inventaire et légendes, Paris : Vuibert, 2005.

Bellamy Chris, Absolute War. Soviet Russia in the Second World War, New York : Knopf, 2008.

Bouchoux Corinne, Rose Valland. La Résistance au musée, La Crêche : Geste éditions, 2006.

Bouchoux Corinne, «Si les tableaux pouvaient parler... » Le traitement politique et médiatique des retours d'œuvres d'art pillées et spoliées par les nazis, France, 1945-2008, Rennes : PUR, 2013.

Brodsky Joseph, «Les trophées », traduit de l'américain par Véronique Schiltz, in Loin de de Byzance, Paris : Fayard, 1988, pp. 417-430.

Brunel Albane, «Le sort du service cinématographique de l'armée en 1940 » in Bertrand Fonck et Amable Sablon du Corail (dir.), 1940, l'empreinte de la défaite. Témoignages et archives, Rennes : PUR, 2014, pp. 77-96.

Cœuré Sophie, La mémoire spoliée. Les archives des Français, butin de guerre nazi puis soviétique (de 1940 à nos jours), Paris : Payot, 2007 (rééd. «Petite Bibliothèque Payot », 2013).

Cœuré Sophie, «Protéger, perdre, oublier ses archives dans la défaite ? France 1940, URSS 1941 » in Gilles Vergnon et Yves Santamaria (dir), Le syndrome de 1940. Un trou noir mémoriel ?, Paris : Riveneuve éditions, 2015, pp. 249-268.

Cœuré Sophie, «Cultural Looting and Restitution at the Dawn of the Cold War : the French Recovery Missions in Eastern Europe ", in Bianca Gaudenzi, Astrid Swenson, MaryAnn Middelkoop (Guest Editors), Journal of Contemporary History, April 2017, Special Issue : The Restitution of Looted Art in 20th-century Europe : Transnational and Global Perspectives (à paraître).

Corcy Stéphanie, La vie culturelle sous l'Occupation, Paris : Perrin, 2005.

Crips Liliane, Nicolas Férard, Nicole Gabriel, «Le cinéma nazi : archives de guerres, archives dispersées par la guerre », Écrire l'histoire, Dossier « Archives », 2014, n 13-14, pp. 119-128.

Dean Martin, «Cultural Looting : the seizure of archives and libraries by Einsatzstab Reichsleiter Rosenberg, 1940-45 », United States Holocaust Memorial Museum, s.d., document en ligne, consulté le 1.02.2017<https://www.ushmm.org/information/exhibitions/online- 
exhibitions/special-focus/offenbach-archival-depot/einsatzstab-reichsleiter-rosenberga-policy-of-plunder>.

Delage Christian, La vérité par l'image. De Nuremberg au procès Milosevic, Paris, Denoël, 2006.

Denis Juliette, «Les archives et l'ennemi. Saisir et protéger les documents soviétiques en Lettonie soviétique (1940-1950) », in Alexandre Sumpf, Vincent Laniol (dir.), Saisies, spoliations et restitutions. Archives et bibliothèques au XXe siècle, Rennes : PUR, 2012, pp. 119-134.

Dmitrieva K., N. Goncharuk, P. Grimsted, et al., Vozvraščenie « Smolenskogo arhiva »: Sbornik statej, Moscou : Rosspen, 2005.

Dreyfus Jean-Marc, Le catalogue Goering, Paris : Flammarion, 2015.

Fomin Valerij, Kino na vojne. Dokumenty i svidetel'stva, Moscou : Materik, 2005.

Gauthier Christophe, Natacha Laurent, «La grande illusion : histoire d'un film », L'Histoire, mars 2012, p. 32.

Grimsted Patricia Kennedy, Reconstructing the Record of Nazi Cultural Plunder : A Guide to the Dispersed Archives of the Einsatzstab Reichsleiter Rosenberg (ERR) and the Postwar Retrieval of ERR Loot, 2016, document en ligne, consulté le 2.02.2017<https://www. errproject.org/guide.php>.

Heuss Anja, Kunst- und Kulturgutraub, Heidelberg : Universitäts Verlag C. Winter, 2000.

Karlsgodt Elizabeth Campbell, Defending National Treasures : French Art and Heritage Under Vichy, Stanford, Calif. : Stanford University Press, 2011.

Kecskeméti Charles, « Saisies d'archives et de bibliothèques : l'évolution du droit international» in A. Sumpf, V. Laniol (dir.), Saisies, spoliations et restitutions. Archives et bibliothèques au XXe siècle, Rennes : PUR, 2012, pp. 25-34.

Kopylova Ol'ga, «K probleme sorhannosti GAF SSSR v gody Velikoj Otečestvennoj Vojny (1941-1945)», Sovetskie Arhivy, 1990, n 5, pp. 37-44.

Kurtz M. J., « The Allied Struggle over Cultural Restitution, 1942-1947 », International Journal of Cultural Property, 2010, n 17, pp. 177-194.

Launey Stéphane, «Les services cinématographiques militaires français pendant la Seconde Guerre mondiale », Revue historique des armées, 2008, $\mathrm{n}^{\circ}$ 252, pp. 27-40 et document en ligne, consulté le 22.02.2017<http://rha.revues.org/3133>.

Leblay-Kinoshita Anne \& Anne-Sophie Maure, « Les leçons de l'année 1940 au ministère des Affaires étrangères » in B. Fonck, A. Sablon du Corail (dir.), 1940, l'empreinte de la défaite. Témoignages et archives, Rennes : PUR, 2014, pp. 105-126.

Mannoni Laurent, Histoire de la cinémathèque française, Paris : Gallimard, 2006.

Moine Nathalie, Les vivants et les morts. Genèse, histoire et héritages de la documentation soviétique des crimes commis en territoires occupés pendant la Seconde Guerre mondiale, fin XIX ${ }^{e}$-début XXI siècle, HDR, Paris : EHESS, 2015.

Naimark Norman, The Russians in Germany. A History of the Soviet Zone of Occupation 19451949, Cambridge, MA : Harvard University Press, 1995.

Pozner Valérie, « Le sort des films trophées saisis par les Soviétiques au cours de la Seconde Guerre mondiale » in A. Sumpf, V. Laniol (dir.), Saisies, spoliations et restitutions. Archives et bibliothèques au XXe siècle, Rennes : PUR, 2012, pp. 147-165.

Pozner Valérie, «L'usage des 'images trophées' dans les films soviétiques » in Valérie Pozner, Alexandre Sumpf, Vanessa Voisin (dir.), Filmer la guerre (1944-1946). Les Soviétiques face à la Shoah, Paris : Éditions du Mémorial de la Shoah, 2015, pp. 91-96. 
Pozner Valérie, Alexandre Sumpf, Vanessa Voisin (dir.), Filmer la guerre (1944-1946). Les Soviétiques face à la Shoah, Paris : Éditions du Mémorial de la Shoah, 2015.

Rousso Henri, Vichy, l'événement, la mémoire, l'histoire, Paris : Gallimard, 2001.

Salomoni Antonella, L'Unione Sovietica e la Shoah. Genocidio, resistenza, rimozione, Bologna : Il Mulino, 2007.

Sandholtz Wayne, "Plunder, Restitution and International Law », International Journal of Cultural Property, 2010, vol. 17, $\mathrm{n}^{\circ}$ 2, Special Issue : Spoils of War v. Cultural Heritage: The Russian Cultural Property Law in Historical Context, pp. 147-176.

Savoy Bénédicte, Patrimoine annexé. Les biens culturels saisis par la France en Allemagne autour de 1800, Paris : Éditions de la Maison des sciences de l'homme, 2003.

Schechter Brandon, Government Issue : The Material Culture of the Red Army 1941-1945, $\mathrm{PhD}$, Berkeley : University of California-Berkeley, 2015.

Stein Wolfgang Hans, «L'idéologie des saisies. Les revendications allemandes des archives, bibliothèques et collections de musées françaises », in A. Sumpf, V. Laniol (dir.), Saisies, spoliations et restitutions. Archives et bibliothèques au XXe siècle, Rennes : PUR, 2012, pp. 67-82.

Vande Winkel Roel, David Welch, Cinema and the Swastika: The International Expansion of Third Reich Cinema, Houndmills, UK : Palgrave MacMillan, 2011.

Voisin Vanessa, L'URSS contre ses traîtres : L'Épuration soviétique (1941-1955), Paris : Publications de la Sorbonne, 2015. 